\title{
Levaduras Termotolerantes: Aplicaciones Industriales, Estrés Oxidativo y Respuesta Antioxidante
}

\author{
Jorge A. Mejía-Barajas, Rocío Montoya-Pérez, Christian Cortés-Rojo, Alfredo Saavedra-Molina* \\ Instituto de Investigaciones Químico-Biológicas. Universidad Michoacana de San Nicolás de Hidalgo. \\ Edificio B-3. C.U. Morelia, Mich. 58030. México. (e-mail: jorge.arturo17@hotmail.com; \\ rmontoya@umich.mx; christiancortesrojo@gmail.com; saavedra@umich.mx)
}

* Autor a quien debe ser dirigida la correspondencia

Recibido Nov. 17, 2015; Aceptado Ene. 13, 2016; Versión final Ene. 27, 2016, Publicado Ago. 2016

\begin{abstract}
Resumen
Se presenta una revisión sobre características y aplicaciones de levaduras termotolerantes, enfocada a aplicaciones industriales y cambios fisiológicos que experimentan las levaduras, con especial énfasis en el estrés oxidativo y la respuesta antioxidante. Las levaduras tienen una amplia aplicación en la biotecnología y son los microorganismos más utilizados en la investigación médica y en la industria. Uno de los usos tradicionales de las levaduras es la fermentación, mediante la cual se pueden obtener bebidas, alimentos y proteínas, entre otros productos. Sin embargo, en este proceso como en otros en los que son utilizadas las levaduras, se generan altas temperaturas que provocan cambios fisiológicos, por lo que se afecta su crecimiento y viabilidad. Lo anterior, ha generado interés en el estudio y desarrollo de levaduras capaces de crecer a temperaturas elevadas (termo tolerantes). Por lo tanto, es de interés caracterizar cepas de levaduras que se utilicen en procesos de fermentación sometidos a altas temperaturas.
\end{abstract}

Palabras claves: levaduras termotolerantes; estrés oxidativo; respuesta antioxidante; biotecnología

\section{Thermotolerant Yeast: Industrial Applications, Oxidative Stress and Antioxidant Response}

\begin{abstract}
A review on characteristics and applications of thermo tolerant yeast, focused on industrial applications and physiological changes that experienced by yeasts, with particular emphasis on oxidative stress and antioxidant response, is presented. Yeasts are widely applied in biotechnology and are the microorganisms most used in medical research and industrial processes. One of the traditional uses of yeast is fermentation, by which beverage, foods and proteins, among others products, can be obtained. However, in this process like in others that use yeasts, high temperatures are generated, causing physiological changes, affecting their growth and viability. This has generated an interest in studying and formulating yeasts capable of growing at elevated temperatures (thermo tolerant yeast). Therefore, it is important to characterize yeast strains to be used in fermentation processes at high temperatures.
\end{abstract}

Keywords: thermo tolerant yeast; applications; oxidative stress; antioxidant response; biotechnology 


\section{INTRODUCCIÓN}

Desde la antigüedad, las levaduras se han reconocido como protagonistas en la producción de alimentos y bebidas mediante la fermentación. Actualmente, son utilizadas en diferentes áreas de la biotecnología, en la tabla 1 se mencionan ejemplos. Aunado a que son un modelo de estudio de células eucariontes las levaduras son los microorganismos más importantes en la biotecnología (Johnson, 2013). Al igual que todos los microorganismos, las levaduras presentan características específicas de acuerdo a la temperatura a la que son cultivadas. Recientemente, se ha generado un creciente interés en las levaduras capaces de crecer a temperaturas elevadas, ya que presentan ventajas en distintos procesos industriales con respecto a las levaduras que no tienen esta cualidad (Koedrit et al., 2008). Las levaduras resistentes a altas temperaturas son denominadas levaduras termotolerantes, sin embargo, no existe un valor absoluto de temperatura, ya que los límites a partir de los cuales se consideran levaduras termotolerantes varían en la literatura. El primer intento por definir levadura termotolerante fue realizado por Arthur y Watson (1976). McCracken y Gong (1982), definen estas levaduras como aquellas con una temperatura de crecimiento máxima de 37 a $45^{\circ} \mathrm{C}$. Koedrith et al. (2008), definieron las levaduras termotolerantes como aquellas capaces de crecer a temperaturas $\geq 40^{\circ} \mathrm{C}$.

Las altas temperaturas generadas durante el uso industrial de levaduras elevan la susceptibilidad de estos microorganismos hacia compuestos inhibitorios del crecimiento (Abdel-Banat et al., 2010), e intensifican el efecto inhibitorio del etanol, por lo que las levaduras termotolerantes podrían presentar una mayor resistencia a estas condiciones. Kwon et al. (2013), reportaron que la cepa termotolerante Isshatchenkia orientalis, presentó una tolerancia de hasta $96.7 \mathrm{gL}^{-1}$ de etanol, siendo el mayor nivel de tolerancia observado en una cepa de levadura crecida a temperaturas de 40 a $45^{\circ} \mathrm{C}$. Las ventajas generales del uso de estas levaduras en los procesos industriales se mencionan en la tabla 2. A continuación, se describen aplicaciones y ejemplos de levaduras termotolerantes en procesos industriales donde presentan ventajas, con respecto a las levaduras que no tienen esta característica, así como una recopilación de los principales cambios fisiológicos que experimentan estas levaduras, enfocado al estrés oxidativo y respuesta antioxidante.

Tabla 1. Industria y usos de las levaduras (Tomado y modificado de Johnson, 2013).

\begin{tabular}{|l|l|}
\hline Fermentaciones tradicionales & Producción de cerveza, vino, sake y salsa de soya \\
\hline Alimentaria & $\begin{array}{l}\text { Producción de enzimas, saborizantes, pigmentos, aminoácidos y ácidos } \\
\text { orgánicos }\end{array}$ \\
\hline Biocatalisis & Estudio de farmacéuticos e intermediarios químicos \\
\hline Biotecnología ambiental & Aplicación en biorremediación y degradación de contaminantes \\
\hline Biocontrol proteínas & Protección de cultivos, alimentos y probióticos \\
\hline $\begin{array}{l}\text { Producción de producción de proteínas farmacéuticas, enzimas, hormonas, vacunas y } \\
\text { toxinas }\end{array}$ \\
\hline Investigación en biología & $\begin{array}{l}\text { Estudio de biología molecular y celular, genómica, vías de ingeniería y } \\
\text { mecanismos de sistemas biológicos }\end{array}$ \\
\hline Investigación biomédica & $\begin{array}{l}\text { Descubrimiento, resistencia y metabolismo de drogas, así como elucidación } \\
\text { de mecanismos de enfermedades }\end{array}$ \\
\hline
\end{tabular}

Tabla 2. Ventajas del uso de levaduras termotolerantes en procesos industriales (Fernández et al., 2008).

\begin{tabular}{|l|}
\hline Reducción de contaminación y costos de enfriamiento. \\
\hline Mayor viabilidad, actividad metabólica y velocidad de fermentación. \\
\hline Mayor mantenimiento de condiciones anaerobias al disminuir la solubilidad del $\mathrm{O}_{2}$. \\
\hline Disminución de la viscosidad del medio de fermentación. \\
\hline Reducción en la formación de subproductos indeseables debido a lisis celular. \\
\hline
\end{tabular}

\section{Producción de biomasa a partir de levaduras termotolerantes}

La producción de biomasa de levaduras, es un proceso económicamente importante y una industria creciente en los últimos años, debido a un aumento en la demanda para las prácticas de la industria vinícola, procesos de manufactura de pan y como complemento en la dieta. La biomasa obtenida de levaduras tiene otras aplicaciones como suplemento proteico en alimentación animal, en la fabricación de ingredientes funcionales y para resaltar el sabor de alimentos procesados (Lee, 1996). El proceso industrial para la producción de biomasa de levaduras, consiste en la propagación de las levaduras de un cultivo en agar a su propagación 
en bioreactores, incrementando el volumen en cada fase de propagación (Di-Serio et al., 2001). Una transformación eficiente de azúcares a biomasa requiere que la producción de metabolitos como etanol y acetaldehído sea minimizada, desviando su metabolismo celular hacia el metabolismo oxidativo, aumentando el rendimiento de la energía en forma de ATP y con esto, la formación de biomasa (Van et al., 1998). Con el objetivo de conservar la biomasa, las levaduras son expuestas a altas temperaturas en un proceso de deshidratación, por ejemplo, la levadura residual de la industria cervecera, la cual después de ser sometida al proceso de deshidratación, es vendida como alimento para animales (Ferreira et al., 2010). Ghorbani et al. (2008), expusieron la biomasa de levadura a altas temperaturas con el objetivo de incrementar la bioabsorción de Cd (cadmio), observando un aumento de hasta $200 \%$. Sin embargo, tanto en este proceso como en la deshidratación para la conservación de biomasa, las moléculas de agua son removidas y las temperaturas incrementan. Lo que afecta la viabilidad y vitalidad de las células (Matthews y Webb, 1991).

La deshidratación genera arresto del ciclo celular y daño a membranas y proteínas (Singh et al., 2005). Por lo anterior, la tolerancia de las levaduras hacia la temperatura es crítica en el mantenimiento de sus componentes celulares y su vitalidad después del proceso de deshidratación. Uno de los principales factores limitantes en la generación de biomasa de levadura, es el alto costo de las fuentes de carbono, por lo que el uso de subproductos agroindustriales es ideal (Zheng et al., 2005). Dichos subproductos deben recibir un tratamiento previo, siendo la exposición a altas temperaturas uno de estos tratamientos. Escalante et al. (1990), analizaron la producción de biomasa por la levadura resistente a temperatura Hansenula polymorpha en un proceso lignocelulósico realizado a $45^{\circ} \mathrm{C}$, observando que es posible obtener mejores resultados al llevarlo a cabo en altas temperaturas. La cepa termotolerante de Kluyveromyces marxianus CBS 712, fue utilizada para la producción de biomasa a partir de suero de leche, un desecho agroindustrial. En los resultados Zoppellari y Bardi, (2013), sugieren el uso de esta cepa para la obtención de biomasa a partir de este desecho, lo que podría reducir los costos de eliminación de efluentes.

\section{Utilización de levaduras termotolerantes como agentes prebióticos y probióticos}

Los prebióticos son ingredientes de alimentos no digestibles que estimulan el crecimiento de bacterias ácido láctico y bifidógenos en el tracto gastrointestinal. Ciertas especies de levaduras, se han utilizado como agentes prebióticos y probióticos para la prevención o el tratamiento de diversos padecimientos intestinales, nutricionales y trastornos toxicológicos (Mosiehi-Jenabian et al., 2010). Los oligosacáridos de la pared celular de las levaduras han demostrado ser un prebiótico de alto valor. Estudios en el uso de las levaduras como probióticos, están relacionados con la generación de levaduras recombinantes con mutaciones en los genes SRB1/PSA1 y PKC1, genes involucrados con la formación de mananos y glucanos de la pared celular. La mutación de estos genes sensibiliza a la levadura a las condiciones del estomago, permitiendo que estas levaduras puedan ser utilizadas como transportadores no solo de probióticos si no además de vacunas terapéuticas (Omara et al., 2010). Algunas levaduras utilizadas con propiedades próbioticas son cepas de Kluyveromyces (Hun et al., 2013) y Saccharomyces (Knoll et al., 2007). La levadura S. cerevisiae es un producto natural de la industria de la cerveza que contiene diferentes compuestos como $\beta$-glucanos, oligosacáridos y ácidos nucleicos. Se ha observado que estos compuestos son capaces de estimular la respuesta inmune (Ortuño et al., 2002).

Por lo anterior S. cerevisiae es utilizada como probiótico en humanos con aplicación oral (Omara et al., 2010). Grobiotec ${ }^{\mathrm{TM}} \mathrm{AE}$, es un prebiótico comercial formado de mezclas de autolisados parciales de levadura de cerveza y productos de fermentación. Celmanax ${ }^{\mathrm{TM}}$ formulado con la pared celular de células de levaduras, actúa como anti adhesivo para la toxina "Shiga" producida por la cepa de Escherichia coli O157:H7, así como micotoxinas. Celmanax ${ }^{\mathrm{TM}}$ también mejora la producción de leche en bovinos y la eficiencia en la conservación de alimentos lácteos (Baines et al., 2011). La levadura S. boulardii actúa como un transportador liberando enzimas, proteínas y factores tróficos durante su tránsito interintestinal, mejorando las defensas inmunológicas del huésped, la digestión y la absorción de nutrientes (Czerucka et al., 2000). S. boulardii también presenta actividad benéfica en la inflamación intestinal mediante la supresión de la activación del NFKB e inhibiendo la expresión de genes de citocinas proinflamatorias (Dalmasso et al., 2006). En el sobrenadante de esta levadura se identificó una molécula estable a temperatura con propiedades antiinflamatorias denominada "factor anti-inflamatorio de Saccharomyces" o "AIF" por sus siglas en ingles (Sougioultzis et al., 2006). Uno de los principales retos para la producción de esta cepa a nivel industrial, es su alta sensibilidad a temperaturas en el proceso de secado. Enshasy (2012), generó en las células de esta levadura tolerancia a la temperatura del proceso de secado, mediante períodos cortos de exposición a altas temperaturas, para posteriormente realizar el secado mediante pulverización, obteniendo mejores resultados. Otra levadura termotolerante la cual se ha sugerido para ser utilizada como probiótico fue aislada de hojas de banana por Koedrith et al. (2008). 


\section{Levaduras termotolerantes en la producción de proteínas recombinantes}

Las levaduras han sido utilizadas desde principios de 1980 para la producción a gran escala de proteínas intracelulares y extracelulares de humanos, animales y plantas (Romanos, 1995). Los sistemas de expresión de proteínas recombinantes basados en levaduras, han demostrado ser una fuente eficiente y económica de proteínas tanto de interés industrial como académico (Mattanovich et al., 2012), convirtiéndose en una de las alternativas más utilizadas para la producción a gran escala. Las levaduras como sistemas de producción de proteínas recombinantes presentan las ventajas de los organismos unicelulares (práctica manipulación genética y rápido crecimiento), así como modificaciones postraduccionales eucariotas. Lo anterior, aunado al mejoramiento de los sistemas de expresión en levaduras, así como la metodología de hibridación, permite un aumento del uso de las levaduras en la producción de proteínas. Algunas de las modificaciones postraduccionales eucariotas que pueden realizar las levaduras, son procesamiento proteolítico, plegamiento, formación de puentes disulfuro y glicosilación (Eckart y Bussineau, 1996). Con respecto a modelos de expresión de proteínas en organismos eucariotas más complejos como células de ovarios de hámster chino y líneas celulares infectadas de baculovirus, las levaduras son más económicas, generalmente presentan mayores rendimientos, no contienen pirógenos y son menos demandantes en términos de tiempo y esfuerzo (Cregg et al., 2000).

Algunas de las desventajas de las levaduras en la producción de proteínas heterólogas, es su incapacidad para realizar ciertas modificaciones postraduccionales como prolil-hidroxilación y amidación (Cregg y Higgins, 1995). La levadura $S$. cerevisiae es una levadura ampliamente utilizada para la producción de proteínas recombinantes de interés industrial o medicinal (Mattanovich et al., 2012). Algunos ejemplos de estas proteínas son la insulina humana, vacunas para virus de hepatitis y del virus del papiloma humano (Martínez et al., 2012). Existen diferentes factores que afectan la producción de proteínas heterólogas en levaduras, algunos de estos son el punto isoeléctrico y la formación de complejos. García-Fruitós et al. (2011) reportaron que la temperatura de crecimiento de las levaduras, es otro factor que afecta el plegado correcto de las proteínas recombinantes, sin embargo, el mecanismo por el cual ocurre esto, aún se desconoce. Recientemente, Zhong et al. (2014), reportaron que la capacidad de plegamiento del retículo endoplasmático (RE) y la viabilidad de la cepa GS115 de Pichia pastoris, son mantenidas a $20^{\circ} \mathrm{C}$, permitiendo altas producciones de la proteína recombinante interleucina-10 de humano (rhlL-10), mientras que a $30^{\circ} \mathrm{C}$ el RE de la levadura sufre de estrés mediante la retención de G3-pro-rhIL10 en un estado inmaduro, dañando la capacidad de plegamiento del RE, disminuyendo la viabilidad celular y producción de la proteína rhIL-10.

Como alternativa a este problema, se ha sugerido que las levaduras termotolerantes podrían producir proteínas termotolerantes, además de poseer ventajas sobre proteínas producidas por otros microorganismos termotolerantes no eucariontes (Takashima et al., 2009). Rodríguez et al. (2000), realizaron un análisis centrado en las diferencias de la proteína recombinante fitasa obtenida en $P$. pastoris, comparada con la proteína obtenida de otros sistemas de expresión, donde se observó que la proteína obtenida de esta levadura, presentó una mayor termotolerancia, conservando el 57 y $40 \%$ de estabilidad a temperaturas de 65 y $90^{\circ} \mathrm{C}$, respectivamente, lo cual es importante, ya que la termotolerancia de la proteína fitasa, es una de las características más deseadas. La levadura Hansenula polimorpha ( $P$. angusta), es una levadura reconocida como termotolerante con capacidad de crecer hasta $49^{\circ} \mathrm{C}$. Esta levadura es ampliamente utilizada para la búsqueda de proteínas termotolerantes (Celik y Calik, 2012). Otra levadura reconocida como termotolerante, aunque menos utilizada para la producción de proteínas heterólogas es Arxula adeninivorans (Wartmann et al., 1995). Aunque existen diversos sistemas de expresión de proteínas heterólogas en levaduras metilotróficas, Promdonkoy et al. (2014), sugirieron que levaduras metilotróficas termotolerantes o termofílicas, pueden ser modelos más adecuados para procesos de producción industrial. El impacto de una gama de temperaturas en la producción de diversas proteínas heterólogas, se ha explorado mediante el uso de la levadura K. marxianus, demostrando ser un sistema práctico para investigar el impacto de la temperatura sobre la eficiencia de la producción de proteínas en levaduras (Raimondi et al., 2013). La levadura K. marxianus es relativamente poco estudiada para la expresión de proteínas heterólogas, en contraste con $S$. cerevisiae y $K$. lactis (Hang et al., 2003). Una mayor comprensión de la fisiología y respuesta a la temperatura en levaduras productoras de proteínas heterólogas, podría permitir la obtención de mayores rendimientos y calidad en la producción de proteínas.

\section{Producción de etanol por levaduras termotolerantes}

El incremento en el uso del etanol por diferentes industrias como fuente de energía, solventes industriales, agentes de limpieza y preservativos, ha incrementado la producción de este alcohol (Brooks, 2008). La producción de etanol puede ser químicamente o a través de la fermentación de carbohidratos, principalmente, por levaduras vía glucólisis en condiciones anaerobias. La producción de etanol mediante la fermentación es un proceso exotérmico, por lo que a medida que la fermentación progresa, el calor generado eleva la temperatura del medio de fermentación (Kumar et al., 2013), además, debido a la corta duración de la fermentación, la refrigeración no siempre es lo suficientemente eficaz en la eliminación de calor, por lo que la 
temperatura puede alcanzar hasta $40^{\circ} \mathrm{C}$ (Andrietta et al., 2002). West y Kennedy, 2014, entre otros, realizaron experimentos con el fin de seleccionar cepas termotolerantes para su uso industrial en la fermentación. Algunas de las ventajas de las levaduras termotolerantes en el proceso de fermentación para la obtención de etanol, son: una mayor tolerancia a compuestos presentes en el medio, ahorro de energía a través de la reducción de costos de refrigeración, mayores tasas de fermentación, separación continua de etanol, reducción del riesgo de contaminación, disminución de la energía requerida para la agitación del medio de fermentación, mayor presión de vapor y aumento de la solubilidad de compuestos orgánicos (Murata et al., 2015).

Por lo anterior, la utilización de cepas de levaduras termotolerantes podría mejorar la eficiencia de la producción de etanol llevando a cabo la fermentación a temperaturas mayores a $40^{\circ} \mathrm{C}$ (Abreu-Cavalheiro y Monteiro, 2013; Caspeta et al., 2014). La levadura termotolerante H. polymorpha es una de las levaduras más estudiadas y la secuencia del genoma completa de la cepa NCY495 es de libre acceso. Se ha observado que cepas de $H$. polymorpha presentan la capacidad de fermentar a altas temperaturas $\left(45\right.$ a $\left.50^{\circ} \mathrm{C}\right) \mathrm{glucosa}$, celobiosa y xilosa a etanol. Kurylenko et al. (2014), generaron una cepa mutante de $H$. polymorpha obteniendo rendimientos de hasta $9.8 \mathrm{~g} / \mathrm{L}$ de etanol, durante la fermentación de xilosa a $45^{\circ} \mathrm{C}$. La producción de etanol a partir de materias primas renovables (bioetanol) representa el proceso de fermentación industrial con mayor crecimiento, con una producción de 85.2 billones de litros de etanol en el 2012 (Renewable fuels association, 2012). La producción de bioetanol es ampliamente investigada como una fuente de combustibles renovables (Vázquez y Dacosta, 2007), debido a que presenta diversas ventajas respecto a los combustibles obtenidos de fósiles. La mayoría de los procesos de obtención de bioetanol a partir de lignocelulosas, comienzan con una hidrólisis termoquímica de la parte hemicelulósica, seguido de una hidrólisis enzimática de la parte celulósica y una fermentación a base de levadura de los azúcares resultantes (Bothast y Schlicher, 2005).

La sacarificación y fermentación simultánea (SFS) es un proceso óptimo para la producción de etanol a partir de fuentes lignocelulósicas. De acuerdo a West y Kennedy (2014), existe una necesidad de realizar investigaciones para el aislamiento de cepas de levaduras termotolerantes que puedan realizar el proceso de SFS a temperaturas mayores de $40^{\circ} \mathrm{C}$. La SFS aumenta los rendimientos de etanol al minimizar la inhibición por producto y eliminar la necesidad de bioreactores separados para ambos procesos (Luo et al., 2008). Uno de los principales inconvenientes del proceso de SFS, son las condiciones de funcionamiento, ya que la hidrólisis enzimática por celulasas tiene una temperatura óptima de 45 a $50^{\circ} \mathrm{C}$, pero la mayoría de las levaduras etanologénicas tienen una temperatura óptima entre 30 y $37^{\circ} \mathrm{C}$ (Taylor et al., 2009). Debido a lo anterior, es que diversos estudios se han centrado en las cepas etanologénicas termotolerantes capaces de realizar la SFS de lignocelulosas (Araque et al., 2008). En la tabla 3, se mencionan estudios en los cuales se utilizaron levaduras termotolerantes y residuos agroindustriales en procesos de SFS para la obtención de etanol, lo que demuestra el potencial de la combinación de estos tres elementos. Castro y Roberto (2014), reportaron que la cepa de K. marxianus NRRL Y-6860, presenta potencial para la producción de etanol mediante la SFS de sustratos celulósicos, ya que presentó un rendimiento de $0.44 \mathrm{~g} / \mathrm{g}$ en la producción de etanol a $45^{\circ} \mathrm{C}$. Moreno et al. (2013), observaron que al remover los fenoles de lignina mediante un tratamiento con lacasa y utilizando la cepa termotolerante de $K$. marxianus CECT 10875, se obtienen rendimientos similares en la producción de etanol, que al utilizar una cepa industrial de $S$. cerevisiae (Fermentis Ethanol Redß, France).

Tabla 3. Producción de bioetanol usando residuos agroindustriales y levaduras termotolerantes en procesos de SFS.

\begin{tabular}{|l|l|l|}
\hline Hojas de maleza Antigonum leptopus & K. fragilis NCIM3358 & Hari et al. (2001). \\
\hline Alcachofa de Jerusalén (Helianthus tuberosus) & $\begin{array}{l}\text { K. marxianus (PT-1) y S. } \\
\text { cerevisae (JZ1C) }\end{array}$ & Hu et al. (2012). \\
\hline Rastrojo de maíz & S. cerevisae DQ1 & Chu et al. (2012). \\
\hline $\begin{array}{l}\text { Subproducto del proceso de extracción del jugo de } \\
\text { zanahoria }\end{array}$ & K. marxianus K21 & Chi-Yang et al. (2013). \\
\hline
\end{tabular}

De acuerdo a Souza et al. (2012), el uso de levaduras termotolerantes y una fase pre-sacarificación antes de realizar la SFS, son claves en el incremento del rendimiento en la producción de bioetanol. Este proceso es conocido como pre-sacarificación y sacarificación y fermentación simultánea (PSFS). Además del proceso de PSFS, se ha investigado el uso de diferentes reactores y condiciones de crecimiento con levaduras termotolerantes para la producción de bioetanol (Lin et al., 2013). La inhibición del crecimiento de las levaduras termotolerantes en el proceso de SFS, debido a los productos de la fermentación de la materia prima lignocelulósica, ha sido estudiada por Wallace-Salinas y Gorwa-Grauslund (2013), desarrollando una cepa clasificada como ISO12, la cual además de la termotolerancia presentó una resistencia a los inhibidores mencionados. 


\section{Principales especies de levaduras termotolerantes}

Las especies de levaduras con más reportes acerca de termotolerancia son $S$. cerevisae y $K$. marxianus, siendo estas últimas las que han mostrado mayor termotolerancia (Nonklang et al., 2008; Suryawati et al., 2008). Las levaduras de K. marxianus son capaces de crecer a temperaturas de hasta $52^{\circ} \mathrm{C}$ (Koedrith et al., 2008), y son una de las especies de levaduras termotolerantes más conocidas y utilizadas. Debido a su alta capacidad de producción de biomasa, etanol, proteínas heterólogas, además de la amplia gama de fuentes de carbono que pueden metabolizar, hacen que esta especie sea atractiva para aplicaciones industriales (Fonseca et al., 2008). Otras de las principales especies de levaduras con termotolerancia, además de las mencionadas, son H. polymorpha (Escalante et al., 1990), P. pastoris (Rodríguez et al., 2000) y K. fragilis (Hari et al., 2001).

\section{Mecanismos de respuesta fisiológicos de las levaduras a altas temperaturas}

La termotolerancia en las levaduras de $S$. cerevisiae puede ser inducida por la exposición a períodos cortos a condiciones estresantes, no letales, tales como bajo $\mathrm{pH}$, alta osmolaridad y concentraciones de etanol, así como temperaturas $\geq$ a $37^{\circ} \mathrm{C}$ (Piper, (1993)). Los efectos letales del estrés por altas temperaturas en levaduras, es el resultado de daño a proteínas, membranas y otras estructuras celulares (Webster y Watson, 1993). Cuando las levaduras se exponen a temperaturas elevadas, exhiben una rápida respuesta molecular, lo que se conoce como la respuesta de choque térmico. La respuesta de choque térmico en $S$. cerevisiae es una de las respuestas más caracterizadas a nivel molecular en células eucariontes. En esta respuesta, se ha observado la expresión de diferentes proteínas, conocidas como proteínas de choque térmico (Hsp's). Mientras que la síntesis de la mayoría de proteínas disminuye a altas temperaturas, la concentración de Hsp's aumenta (Parsell y Lindquist, 1994). La proteína Hsp104 está involucrada en los cambios estructurales en proteínas y complejos proteicos (Cashikar et al., 2005). La proteína Hsp104 de S. cerevisiae restaura la estructura de proteínas desnaturalizadas usando energía en forma de ATP y un sistema de chaperonas Hsp70p/Hsp40p (Weibezahn et al., 2004). La expresión de la Hsp104 en S. cerevisiae es baja en condiciones normales de temperatura, sin embargo, a altas temperaturas aumenta significativamente su concentración. La expresión del gen de la proteína Hsp104 provee a S. cerevisiae de termotolerancia (Lindquist y Kim, 1996). Además de la síntesis transitoria de Hsp's, la respuesta a altas temperaturas, consiste en cambios en la composición lipídica de las membranas (Benschoter e Ingram, 1986).

En una investigación reciente, se demostró que cepas de $S$. cerevisiae termotolerantes aisladas mediante evolución adaptativa a temperaturas de $40^{\circ} \mathrm{C}$ o mayores, modificaron la composición de esteroles de sus membranas de ergosterol a fecosterol. Lo anterior, causado por una mutación en el gen de la desaturasa esterol C-5 y a un incremento en la expresión de genes relacionados con la biosíntesis de esteroles. Se ha sugerido que la alteración en la composición de esteroles de las cepas adaptadas, resulta en una fluidez óptima de las membranas permitiendo que estas levaduras sobrevivan (Caspeta et al., 2014). Sotamura et al. (2013), generaron la cepa termotolerante YK60-1, a partir de la cepa de S. cerevisiae MT8-1, mediante la exposición a temperaturas con aumento progresivo, e investigaron mediante análisis de microarreglos de ADN el mecanismo mediante el cual, la cepa MT8-1 adquirió termotolerancia. En sus resultados, observaron la inducción de genes de respuesta a estrés, tales como los de Hsp y biosíntesis de trehalosa. Mediante análisis de metaboloma, mostraron que la cepa YK60-1 acumuló mayor concentración de trehalosa.

Originalmente, se creía que la trehalosa funcionaba como un carbohidrato de almacenamiento en las levaduras, pero se ha observado que puede ser un protector de la tensión relacionada con la termotolerancia inducida. La trehalosa es un carbohidrato protector que se acumula después de la exposición a altas temperaturas (Mahmud et al., 2010; An et al., 2011). Virgilio et al. (1994), proporcionaron evidencia genética de que la síntesis de trehalosa inducida por altas temperaturas es un factor importante para la inducción de la termotolerancia. La sobre expresión del gen TPS1 que convierte la glucosa 6-fostato y glucosa-UDP en trehalosa 6-fosfato, genera un aumento en la concentración de trehalosa, por lo que TPS1 está involucrado en la tolerancia a la temperatura (An et al., 2011). Recientemente, Petitjean et al. (2015), utilizando una combinación de mutantes de levaduras en el gen Tps1 y cepas de levaduras capaces de asimilar trehalosa de una fuente externa, proporcionaron evidencia de que la trehalosa no protege las células, identificando la proteína Tsp1 con una función clave para la resistencia a la temperatura, posiblemente a través del mantenimiento de los niveles de ATP. Otros factores fisiológicos implicados en la termotolerancia, incluyen el agua celular no congelable (Komatsu et al., 1991), la detención del ciclo celular en la fase Go (Plesset et al., 1987), la fosforilación de proteínas independiente de AMPc y de la ATPasa unida a membrana (Coote et al., 1994). La tolerancia del metabolismo de las levaduras a alta temperatura es controlada por diferentes genes, los cuales están relacionados con la síntesis o acumulación de metabolitos específicos para la protección de las células en altas temperaturas, sin embargo, en cuanto a la genómica que podría estar relacionada con la termotolerancia de las levaduras, poco se conoce (Abreu-Cavalheiro y Monteiro (2013)). 
En un trabajo realizado por Krsmanović y Kölling (2004), se describió una cepa de levadura con la capacidad de crecer a altas temperaturas, la cual presenta un alelo conocido como RSP5. RSP5 tiene una función en el transporte celular y degradación de proteínas. Levaduras mutadas en RSP5 perdieron la habilidad para acumular conjugados de ubiquinona, después de ser expuestas a choque térmico, por lo que se sugirió que RSP5 está relacionado con la sensibilidad a la temperatura. En las levaduras en las que se aumentó los niveles de la transcripción del gen RSP5-C, se observó un aumento en la ubiquitinación de proteínas, así como en la tolerancia a la temperatura (Shahsavarani et al., 2011). En otro estudio, en el que se clonó el gen Hsp100 de Pleurotus sajor-caju en una levadura, se observó un aumento en la sobrevivencia de las levaduras expuestas a $50^{\circ} \mathrm{C}$ (Lee et al., 2006). Kim et al. (2011), reportaron que la inhibición de los genes SSK2, PPG1 y PAM1, generan una reducción en la tolerancia a la temperatura. Más reciente, Caspeta et al. (2014), secuenciaron el genoma de 7 cepas de levaduras termotolerantes generadas mediante evolución adaptativa de la cepa CEN.PK113-7D, la cual, fue expuesta a temperatura de $39^{\circ} \mathrm{C}$ hasta por 90 días. La secuenciación del genoma de las levaduras reveló un total de 30 variaciones de nucleótidos simples en 18 genes. La mayoría de las variaciones fueron detectadas en genes que afectan la composición y estructura de la membrana, respiración, replicación y reparación del ADN. Además de lo mencionado, se ha reportado que el estrés por temperatura induce una hiperpolarización o desporalización (Balogh et al., 2005) mitocondrial, causando probablemente, una subsecuente producción de especies reactivas de oxígeno (ERO) (Perl et al., 2004) y estrés oxidativo.

\section{Estrés oxidativo en levaduras generado por altas temperaturas}

El estrés oxidativo generado por la exposición a altas temperaturas, es uno de los principales factores en la muerte de S. cerevisiae (Davidson y Schiestl, 2001). Las ERO generadas a altas temperaturas, alteran las membranas celulares, proteínas y ADN, y causan finalmente, la muerte celular (Storz y Imlay, 1999). Sugiyama et al. (2000), observaron que independiente del tipo de metabolismo de las levaduras (oxidativo o fermentativo), se presenta un incremento en los niveles de ERO conforme aumenta la temperatura. El estrés por temperatura puede inducir diferentes genes antioxidantes en levaduras, mientras que el $\mathrm{H}_{2} \mathrm{O}_{2}$ induce diferentes Hsp's. Lo anterior muestra una relación entre el estrés por temperatura y el estrés oxidativo, por lo cual, se ha sugerido que las Hsp's y los sistemas antioxidantes, contribuyen a la termotolerancia intrínseca de las levaduras (Morano et al., 2012). Rikhvanov et al. (2001), observaron que la exposición de levaduras a $45^{\circ} \mathrm{C}$ estimula su respiración, lo cual podría incrementar la generación de ERO y eventualmente causar la muerte celular, mientras que la represión de su respiración celular permite una mayor tolerancia a temperaturas de $45^{\circ} \mathrm{C}$.

Mutantes de levaduras con deleción en genes para enzimas antioxidantes como catalasa, superóxido dismutasa y tiorredoxina peroxidasa, son más sensibles al efecto letal de altas temperaturas, mientras que la sobreexpresión de los genes de la catalasa y la superóxido dismutasa, causa un aumento en su termotolerancia (Davidson y Schiestl, 2001). Caspeta et al. (2014), reportaron que cepas de levaduras termotolerantes generadas mediante exposición a $39^{\circ} \mathrm{C}$, fueron incapaces de metabolizar fuentes de carbono no fermentables y no mostraron cambio diáuxico a 40 y $30^{\circ} \mathrm{C}$. Estas cepas acumularon mutaciones en los genes ATP2 o ATP3, los cuales son esenciales para el crecimiento en fuentes de carbono no fermentables. A lo anterior, sugieren que el fenotipo óptimo de levaduras termotolerantes podría no ser a través de la evolución, mientras exista una respiración oxidativa totalmente funcional, posiblemente, debido a que esto generaría una mayor concentración de ERO induciendo estrés oxidativo.

Recientemente, Cao et al. (2013), reportaron una asociación en el incremento de la producción de intermediarios de especies reactivas de oxígeno (IERO), con la vía de la lanzadera de GABA, cuando las levaduras fueron expuestas a choque térmico. Esta vía contiene las enzimas glutamato descarboxilasa (GAD), GABA aminotransferasa (GAT) y succinato semialdehído deshidrogenasa (SSADH). En levaduras, esta vía está relacionada con la formación de succinato a partir de a-cetoglutarato, la cual provee carbono en la cadena respiratoria en la mitocondria. En su trabajo, Cao et al. (2013), concluyen que el daño en las células de levadura producido por la exposición a altas temperaturas, es prevenido por la función de las enzimas de la lanzadera GABA, a través de un mecanismo que se relaciona con el abatimiento de la acumulación de IERO durante el estrés térmico. Se ha observado esta relación, entre la producción de ERO y la termotolerancia, ya que en nuestro grupo de investigación, se determinó la concentración de ERO generadas en tres cepas de levaduras, las cuales fueron aisladas de zonas productoras de mezcal en México, de los estados de Oaxaca, Guerrero y San Luis Potosí. Estos lugares son típicos de tener variaciones en la temperatura, sobretodo, altas temperaturas (Arellano-Plaza et al., 2013).

\section{CONCLUSIONES}

Entre las principales características que tienen las levaduras termotolerantes, se pueden mencionar las siguientes: 
i) La termotolerancia de las levaduras es una propiedad importante en aplicaciones industriales.

ii) La respuesta antioxidante es un factor importante en la termotolerancia, sin embargo, es necesario ampliar el conocimiento del efecto de la temperatura en esta respuesta, con posibles implicaciones en el uso industrial de las levaduras.

\section{AGRADECIMIENTOS}

Se agradece el apoyo para el desarrollo de este trabajo de CONACYT (169093, ASM); así como el apoyo de la CIC-UMSNH (2015, ASM). JAMB es becario de CONACYT.

\section{REFERENCIAS}

Abdel-Banat, B.M.A., H. Hoshida, A. Ano, H. Hoshida, S. Nonklang y R. Akada, High-temperature fermentation: how can processes for ethanol production at high temperatures become superior to the traditional process using mesophilic yeast?, doi: 10.1007/s00253-009-2248-5, Appl Microbiol Biotechnol, 85(4), 861-867 (2010)

Abreu-Cavalheiro, A. y G, Monteiro, Solving ethanol production problems with genetically modified yeast strains, Braz J Microbiol, 44(3), 665-671 (2013)

An, M.Z., Y.Q. Tang, K. Mitsumasu, Z.S. Liu, M. Shigeru y K. Kenji, Enhanced thermotolerance for ethanol fermentation of Saccharomyces cerevisiae strain by overexpression of the gene coding for trehalose-6phosphate synthase, doi: 10.1007/s10529-011-0576-x, Biotechnol Lett, 33(7), 1367-1374 (2011)

Andrietta, M.G.S., S.R. Andrietta, C. Steckelberg y E.N.A. Stupiello, Bioethanol - 30 years after Proálcool, Int Sugar J, 109(1299), 195-200 (2002)

Araque, E., C. Parra, M., Rodríguez, J., Freer y J. Baeza, Selection of thermotolerant yeast strains Saccharomyces cerevisiae for bioethanol production, doi:10.1016/j.enzmictec.2008.02.007, Enzyme Microb Tech, 43(2), 120-123 (2008)

Arellano-Plaza, M., A. Gschaedler-Mathis, R. Noriega-Cisneros, M. Clemente-Guerrero, S. Manzo-Ávalos, J.C. González-Hernández y A. Saavedra-Molina, Respiratory capacity of the Kluyveromyces marxianus yeast isolated from the mezcal process during oxidative stress, doi: 10.1007/s11274-013-1291-7, World J Microbiol Biotechnol, 29(7), 1279-1287 (2013)

Arthur, $\mathrm{H}$ y K. Watson, Thermal adaptation in yeast: growth temperatures, membrane lipid, and cytochrome composition of psychrophilic, mesophilic, and thermophilic yeasts, J Bacteriol, 128(1), 58-68 (1976)

Baines, D., S. Erb, K. Turkington, G. Kuldau, J. Juba, L. Masson, A. Mazza y R. Roberts, Mouldy feed, mycotoxins and Shiga toxin - producing Escherichia coli colonization associated with Jejunal Hemorrhage Syndrome in beef cattle, doi: 10.1186/1746-6148-7-24, BMC Vet Res, 7,24 (2011)

Balogh, G., I. Horváth, E. Nagy, Z. Hoyk, S. Benkõ, O. Bensaude y L. Vígh, The hyperfluidization of mammalian cell membranes acts as a signal to initiate the heat shock protein response, doi: $10.1111 / \mathrm{j} .1742$ 4658.2005.04999.x, FEBS J, 272(23), 6077-6086 (2005)

Benschoter, A.S y L.O. Ingram, Thermal tolerance of Zymomonas mobilis: Temperature-induced changes in membrane composition, Appl Environ Microbiol, 51(6), 1278-1282 (1986)

Bothast, R.J y M.A. Schlicher, Biotechnological processes for conversion of corn into ethanol, doi: 10.1007/s00253-004-1819-8, Appl Microbiol Biotechnol, 67(1), 19-25 (2005)

Brooks, A.A., Ethanol production potential of local yeast strains isolated from ripe banana peels. African $\mathrm{J}$ Biotechnol, 7(20), 3749-3752 (2008)

Cao, J., J.M. Barbosa, N.K. Singh y R.D. Locy, GABA shunt mediates thermotolerance in Saccharomyces cerevisiae by reducing reactive oxygen production, doi: 10.1002/yea.2948, Yeast, 30(4), 129-144 (2013)

Cashikar, A.G., M. Duennwald y S.L. Lindquist, A chaperone pathway in protein disaggregation. Hsp26 alters the nature of protein aggregates to facilitate reactivation by Hsp104, doi:10.1074/jbc.M502854200, J Biol Chem 280(25), 23869-23875 (2005) 
Caspeta, L., Y. Chen, P. Ghiaci, A. Feizi, S. Buskov, B.M. Hallström, D. Petranovic y J. Nielsen, Biofuels Altered sterol composition renders yeast thermotolerant, doi: 10.1126/s, Science, 346(6205), 75-78 (2014)

Castro, R.C., y I.C. Roberto, Selection of a thermotolerant Kluyveromyces marxianus strain with potential application for cellulosic ethanol production by simultaneous saccharification and fermentation, doi: 10.1007/s12010-013-0612-5, Appl Biochem Biotechnol, 172(3), 1553-1564 (2014)

Celik, E., y P. Calik, Production of recombinant proteins by yeast cells, doi: 10.1016/j, Biotechnol Adv, 30(5), 1108-1118 (2012)

Chi-Yang, Y., J. Bo-Hong y D. Kow-Jen, Production of bioethanol from carrot pomace using the thermotolerant yeast Kluyveromyces marxianus, doi: 10.3390/en6031794, Energies, 6(3), 1794-1801 (2013)

Chu, D., J. Zhang y J. Bao, Simultaneous saccharification and ethanol fermentation of corn stover at high temperature and high solids loading by a thermotolerant strain Saccharomyces cerevisiae DQ1, doi: 10.1007/s12155-012-9219-x, Bioenerg Res, 5(4), 1020-1026 (2012)

Coote, P J., M.V. Jones, I.J. Seymour, D.L. Rowe, D.P. Ferdinando, A.J. McArthur, y M.B. Cole, Activity of the plasma membrane $\mathrm{H}+$-ATPase is a key physiological determinant of thermotolerance in Saccharomyces cerevisiae, Microbiology 140, 1881-1890 (1994)

Cregg, J.M., J.L. Cereghino, J. Shi y D.R. Higgins, Recombinant protein expression in Pichia pastoris, doi: 10.1385/MB:16:1:23, Mol Biotechnol, 16(1), 23-52 (2000)

Cregg, J.M y D.R. Higgins, Production of foreign proteins in the yeast Pichia pastoris, doi: 10.1139/b95-336, Can J Bot, 73, 891-897 (1995)

Czerucka, D., S. Dahan, B. Mograbi, B. Rossi y P. Rampal, Saccharomyces boulardii preserves the barrier function and modulates the signal transduction pathway induced in enteropathogenic Escherichia coli-infected T84 cells, doi: 10.1128/IAI.68.10.5998-6004.2000, Infect Immun, 68(10), 5998-6004 (2000)

Dalmasso, G., F. Cottrez, V. Imbert, P. Lagadec, J.F. Peyron, P. Rampal, D. Czerucka, H. Groux, A. Foussat y $V$. Brun, Saccharomyces boulardii inhibits inflammatory bowel disease by trapping $T$ cells in mesenteric lymph nodes, http://dx.doi.org/10.1053/j.gastro.2006.10.001, Gastroenterology, 131(6), 1812-25 (2006)

Davidson, J.F., y R.H. Schiestl, Cytotoxic and genotoxic consequences of heat stress are dependent on the presence of oxygen in Saccharomyces cerevisiae, doi: 10.1128/JB.183.15.4580-4587.2001, J Bacteriol, 183(15), 4580-4587 (2001)

Souza, C.J., D.A. Costa, M.Q. Rodrigues., A.F. dos Santos, M.R. Lopes., A.B. Abrantes, P. dos Santos Costa, W.B. Silveira., F.M. Passos y L.G. Fietto, The influence of presaccharification, fermentation temperature and yeast strain on ethanol production from sugarcane bagasse, doi: 10.1016/j.biortech.2012.01.024, Bioresour Technol, 109, 63-69 (2012)

Di-Serio, M., R. Tesser y E. Santacesaria, A kinetic and mass transfer model to simulate the growth of baker's yeast in industrial bioreactors, doi: 10.1016/S0920-5861(00)00644-1, Chem Eng J, 82, 347-354 (2001)

Eckart, M.R y C.M., Bussineau, Quality and authenticity of heterologous proteins synthesized in yeast, doi: 10.1016/S0958-1669(96)80056-5, Curr Opin Biotechnol, 7(5), 525-530 (1996)

Enshasy, H., Industrial platform design for large scale production of probiotic yeast. Paper No. 531c. 12 AIChE, Annual meeting, Pittsburgh, USA.ISBN: 978-0-8169-1073-1 (2012)

Escalante, J., G. Caminal y C. de Mas, Biomass production by a thermotolerant yeast: Hansenula polymorpha, doi: 10.1002/jctb.280480106, J Chem Technol Biotechnol, 48(1), $61-70$ (1990)

Ferreira, I.M.P.L.V.O., O. Pinho, E. Vieira y J.G. Tavarela, Brewer's Saccharomyces yeast biomass: characteristics and potential applications, doi:10.1016/j.tifs.2009.10.008, Trends Food Sci Technol, 21, 77-84 (2010)

Fernández, T., M. Marcet, W. Olivera y C. Martín, Aislamiento y evaluación de cepas termotolerantes de Saccharomyces cerevisiae para la producción de aguardientes y rones. Cienc. Tecnol. Aliment. 6(1), 64-70 (2008) 
Fonseca, G.G., E. Heinzle, C. Wittmann y A.K. Gombert, The yeast Kluyveromyces marxianus and its biotechnological potential, doi: 10.1007/s00253-008-1458-6, Appl Microbiol Biotechnol, 79(3), $339-354$ (2008)

García-Fruitós, E., E. Vazquez, N. Gonzalez-Montalbán, N. Ferrer-Miralles y A. Villaverde, Analytical approaches for assessing aggregation of protein biopharmaceuticals, doi: http://dx.doi.org/10.2174/138920111798357339, Curr Pharm Biotechnol, 12(10), 1530-1536 (2011)

Ghorbani, F., H. Younesi, S.M. Ghasempouri, A.A. Zinatizadeh, M. Amini y A. Daneshi, Application of response surface methodology for optimization of cadmium biosorption in an aqueous solution by Saccharomyces cerevisiae, doi:10.1016/j.cej.2008.04.028, Chem Eng J, 145, 267- 275 (2008)

Hang, Y.D., E.E. Woodams y L.E. Hang, Utilization of corn silage juice by Kluyveromyces marxianus, Bioresour Technol, 86(3), 305-307 (2003)

Hari, K.S., Janardhan R.T., Chowdary, G.V., Simultaneous saccharification and fermentation of lignocellulosic wastes to ethanol using thermotolerant yeast, doi:10.1016/S0960-8524(00)00151-6, Bioresour Technol 77:193- 196 (2001)

Hu, N., B. Yuan, J. Sun, S.A. Wang y F.L. Li, Thermotolerant Kluyveromyces marxianus and Saccharomyces cerevisiae strains representing potentials for bioethanol production from Jerusalem artichoke by consolidated bioprocessing, doi: 10.1007/s00253-012-4240-8, Appl Microbiol Biotechnol, 95(5), 1359-1368 (2012)

Hun, C.H., M.S. Mohd Sued, R. Abd Malek, Z. Othman, E.A. Elsayed, S. Ramili, N.A. Elmarzugi, M.R. Sarmidi, R. Aziz y H.A. Enshasy, Bioprocess development for high cell mass production of the probiotic yeastKluyveromyces lactis, doi: 10.9790/3008-0834959, IOSR J Pharm Biol Sci, 8, 49-59 (2013)

Johnson, E.A., Biotechnology of non-Saccharomyces yeasts-the basidiomycetes, doi: 10.1007/s00253-0135046-z, Appl Microbiol Biotechnol, 97(17), 503-517 (2013)

Kim, H.S., N.R. Kim, J. Yang y W. Choi, Identification of novel genes responsible for ethanol and/or thermotolerance by transposon mutagenesis in Saccharomyces cerevisiae, doi: 10.1007/s00253-011-3298-z, Appl Microbiol Biotechnol, 91(4), 1159-1172 (2011)

Koedrith, P., E. Dubois, B. Scherens, E. Jacobs, C. Boonchird y F. Messenguy, Identification and characterization of a thermotolerant yeast strain isolated from banana leaves, doi: 10.2306/scienceasia15131874.2008.34.147, Science Asia, 34, 147-152 (2008)

Komatsu, Y., K. Obuchi, H. Iwahashi, S.C. Kaul, M. Ishimura, G.M. Fahy y W.F. Rall, Deuterium oxide, dimethylsulfoxide and heat shock confer protection against hydrostatic pressure damage in yeast, doi: 10.1016/0006-291X(91)91539-O, Biochem Biophys Res Commun, 174(3), 1141-1147 (1991)

Knoll, A., S. Bartsch, B. Husemann, P. Engel, K. Schroer, B. Ribeiro, C. Stöckmann, J. Seletzky y J. Büchs, High cell density cultivation of recombinant yeasts and bacteria under non-pressurized and pressurized conditions in stirred tank bioreactors, doi:10.1016/j.jbiotec.2007.06.010, J Biotechnol, 132(2), 167-179 (2007)

Krsmanović, T y R. Kölling, The HECT E3 ubiquitin ligase Rsp5 is important for ubiquitin homeostasis in yeast, doi: http://dx.doi.org/10.1016/j.febslet.2004.10.006, FEBS Lett, 577(1-2), 215-219 (2004)

Kumar S., P. Dheeran, P. Surendra, I.M. Mishra, D.K. Adhikari, Cooling system economy in ethanol production using thermotolerant yeast Kluyveromyces Sp. IIPE453, doi: 10.12691/ajmr-1-3-1, Am J Microbiol Res, 1(3), 39-44 (2013)

Kurylenko, O.O., J. Ruchala, O.B. Hryniv, C.A. Abbas, K.V. Dmytruk y A.A. Sibirny, Metabolic engineering and classical selection of the methylotrophic thermotolerant yeast Hansenula polymorpha for improvement of hightemperature xylose alcoholic fermentation, doi: 10.1186/s12934-014-0122-3, Microbial Cell Fact, 13, 122 (2014)

Kwon, Y.J., F. Wang, Q. Li, L. C.Z. Li, Effect of temperature on ethanol tolerance of thermotolerant Isshatchenkia orientalis IPE100, doi: 10.1002/elsc.201100205, Eng Life Sci, 13(2), 126-131 (2013)

Lee, B., Fundamentos de biotecnología de los alimentos, Zaragoza, España, Editorial Acribia, 77-89 (1996)

Lee, J.O., M.J. Jeong, T.R. Kwon, S.K. Lee, M.O. Byun, I.M. Chung y S.C. Park, Pleurotus sajor-caju HSP100 complements a thermotolerance defect in hsp104 mutant Saccharomyces cerevisiae, J Biosci, 31(2), 223-233 (2006) 
Lin, Y.S., W.C. Lee, K.J. Duan y Y.H. Lin, Ethanol production by simultaneous saccharification and fermentation in rotary drum reactor using thermotolerant Kluveromyces marxianus, doi:10.1016/j.apenergy.2012.12.020, Appl Ener, 105, 389-394 (2013)

Lindquist, S y G. Kim, Heat_shock protein 104 expression is sufficient for thermotolerance in yeast. Proc Natl Acad Sci USA 93(11), 5301-5306 (1996)

Luo, P., Z. Liu, C. Yang y G. Wang, Study of simultaneous saccharification and fermentation for steam exploded wheat straw to ethanol, doi: 10.1007/s11705-008-0069-1, Front Chem Eng China 2, 447-451 (2008)

Mahmud, S.A., T. Hirasawa y H. Shimizu, Differential importance of trehalose accumulation in Saccharomyces cerevisiae in response to various environmental stresses, doi: 10.1016/j.jbiosc.2009.08.500, J Biosci Bioeng, 109(3), 262-266 (2010)

Martinez, J.L., L. Liu, D. Petranovic y J. Nielsen, Pharmaceutical protein production by yeast: towards production of human blood proteins by microbial fermentation, doi: 10.1016/j.copbio.2012.03.011, Curr Opin Biotechnol, 23,1-7 (2012)

Mattanovich, D., P. Branduardi, L. Dato, B. Gasser, M. Sauer y D. Porro, Recombinant protein production in yeasts, doi: 10.1007/978-1-61779-433-9_17, Methods Mol. Biol, 824, 329-358 (2012)

Matthews, T.M y C. Webb, Culture systems, In: Biotechnolgy Handbooks 4: Saccharomyces (Tuite, M.F., Oliver, S.G., Eds.) 249-282. Plenum Press (1991)

McCracken, L.D y C.S. Gong, Fermentation of cellulose and hemicellulose carbohydrates by thermotolerant yeasts, Biotechnol Bioeng, 12, 91-102 (1982)

Morano, K.A., C.M. Grant, y W.S. Moye-Rowley, The Response to Heat Shock and Oxidative Stress in Saccharomyces cerevisiae, doi: 10.1534/genetics.111.128033, Genetics, 190(4), 1157-1195 (2012)

Moreno, A.D., D. Ibarra, I. Ballesteros, A. González y M. Ballesteros, Comparing cell viability and ethanol fermentation of the thermotolerant yeast Kluyveromyces marxianus and Saccharomyces cerevisiae on steamexploded biomass treated with laccase, doi: 10.1016/j.biortech.2012.11.095, Bioresour Technol, 135, 239-245 (2013)

Mosiehi-Jenabian, S., L.L. Pedersen y L. Jespersen, Beneficial effects of probiotic and food borne yeasts on human health, doi: 10.3390/nu2040449, Nutrients, 2(4), 449-473 (2010)

Murata, M., Nitiyon, S., Lertwattanasakul, N., Sootsuwan, K., Kosaka, T., Thanonkeo, P., Limtong, S., Yamada, M., High-temperature Fermentation Technology for Low-cost Bioethanol, doi: http://doi.org/10.3775/jie.94.1154, Journal of the Japan Institute of Energy, 94 (10), 1154-1162 (2015)

Nonklang, S., B.M. Abdel-Banat, K. Cha-aim, N. Moonjai, H. Hoshida, S. Limtong, M. Yamada y R. Akada, High-temperature ethanol fermentation and transformation with linear DNA in the thermotolerant yeast Kluyveromyces marxianus DMKU3-1042, doi: 10.1128/AEM.01854-08, Appl Environ Microbiol, 74(24), 75147521 (2008)

Omara, W.A., B.M. Rash, A. Hayes, M.S. Wickham, S.G. Oliver y L.I. Stateva, Conditional cell-wall mutants of Saccharomyces cerevisiae as delivery vehicles for therapeutic agents in vivo to the Gl tract, doi:10.1016/j.jbiotec.2010.03.010, J Biotechnol, 147(2), 136-143 (2010)

Ortuño, J., A. Cuesta, A. Rodríguez, M.A. Esteban y J. Meseguer, Oral administration of yeast, Saccharomyces cerevisiae, enhances the cellular innate immune response of gilthead seabream (Sparus aurata L, doi:10.1016/S0165-2427(01)00406-8, Vet Immunol Immunopathol, 85, 41-50 (2002)

Parsell, D.A y S. Lindquist, Heat shock proteins and stress tolerance. doi: 10.1016/S0959-437X(05)80135-2 In The Biology of Heat Shock Proteins and Molecular Chaperones. New York: Cold Spring Harbor. 457-489 (1994)

Perl, A., P.J. Gergely, G. Nagy, A. Koncz y K. Banki, Mitochondrial hyperpolarization: a checkpoint of T-cell life, death and autoimmunity, doi: 10.1016/j.it.2004.05.001, Trends Immunol, 25(7), 360-367 (2004)

Petitjean, M., M.A. Teste, J.M., Francois y J.L. Parrou, Yeast tolerance to various stresses relies on the trehalose-6P synthase (Tps1) protein, not on trehalosa, doi: 10.1074/jbc.M115.653899, The Journal of Biological Chemistry, 290(26), 16177-16190 (2015) 
Piper, P.W. Molecular events associated with acquisition of heat tolerance by the yeast Saccharomyces cerevisiae, doi: http://dx.doi.org/10.1111/j.1574-6976.1993.tb00005.x, FEMS Microbiol Rev, 11(4), 339-355 (1993)

Plesset, J., J.R. Ludwig, B.S. Cox y C.S. McLaughlin, Effect of cell cycle position on thermotolerance in Saccharomyces cerevisiae, J Bacteriol, 169(2), 779-784 (1987)

Promdonkoy, P., W. Tirasophon, N. Roongsawang, L. Eurwilaichitr y S. Tanapongpipat, Methanol-inducible promoter of thermotolerant methylotrophic yeast Ogataea thermomethanolica BCC16875 potential for production of heterologous protein at high temperatures, doi: 10.1007/s00284-014-0568-x, Curr Microbiol, $69(2), 143-148(2014)$

Raimondi, S., E. Zanni, A. Amaretti, C. Palleschi, D. Uccelletti y M. Rossi, Thermal adaptability of Kluyveromyces marxianus in recombinant protein production, doi: 10.1186/1475-2859-12-34, Microb Cell Fact, 12,34 (2013)

Renewable fuels association. [http://www.ethanolrfa.org/news/entry/global-ethanol-production-to-reach-85.2billion-litres-in-2012]

Rikhvanov, E.G., N.N. Varakina, T.M. Rusaleva, E.I. Rachenko, V.A. Kiseleva y V.K. Voinikov, Heat shockinduced changes in the respiration of the yeast Saccharomyces cerevisiae, doi: 10.1023/A:1010442429489, Mikrobiolgiia, 70(4), 531-535 (2001)

Rodríguez, E., E.J. Mullaney y X.G. Lei, Expression of the Aspergillus fumigatus phytase gene in Pichia pastoris and characterization of the recombinant enzyme, doi: 10.1006/bbrc.2000.2121, Biochem Biophys Res Commun, 268(2), 373-378 (2000)

Romanos, M. Advances in the use of Pichia pastoris for high-level gene expression, doi: 10.1016/09581669(95)80087-5, Curr Opin Biotech, 6, 527-533 (1995)

Shahsavarani, H., M. Sugiyama, Y. Kaneko, B. Chuenchit y S. Harashima, Superior thermotolerance of Saccharomyces cerevisiae for efficient bioethanol fermentation can be achieved by overexpression of RSP5 ubiquitin ligase, doi: 10.1016/j.biotechadv.2011.09.002, Biotechnol Adv, 30(6), 1289-1300 (2011)

Singh, J., D. Kumar, N. Ramakrishnan, V. Singhal, J. Jervis, J.F. Garst, S.M. Slaughter, A.M. DeSantis, M. Potts y R.F. Helm, Transcriptional response of Saccharomyces cerevisiae to desiccation and rehydration, doi: 10.1128/AEM.71.12.8752-8763.2005, Appl Environ Microbiol, 71(12), 8752-8763 (2005)

Sotamura, A., Y. Katsuyama, N. Miura, K. Kuroda, A. Tomio, T. Bamba, E. Fukusaki y M. Ueda, Acquisition of thermotolerant yeast Saccharomyces cerevisiae by breeding via stepwise adaptation, doi: 10.1002/btpr.1754, Biotechnol Prog, 29(5), 1116-1123 (2013)

Sougioultzis, S., S. Simeonidis, K.R. Bhaskar, X. Chen, P.M. Anton, S. Keates, C. Pothoulakis y C.P. Kelly. Saccharomyces boulardii produces a soluble anti-inflammatory factor that inhibits NF-kappaB-mediated IL-8 gene expression, doi:10.1016/j.bbrc.2006.02.080, Biochem Biophys Res Commun, 343(1), 69-76 (2006)

Sugiyama, K., S. Izawa y Y. Inoue, The Yap1p-dependent induction of glutathione synthesis in the heat shock response of Saccharomyces cerevisiae, doi: 10.1074/jbc.275.20.15535, J Biol Chem, 275(20), 15535-15540 (2000)

Suryawati, L., M.R. Wilkins, D.D. Bellmer, R.L. Huhnke, N.O. Maness y I.M. Banat, Simultaneous saccharification and fermentation of Kanlow switchgrass pretreated by hydrothermolysis using Kluyveromyces marxianus IMB4, doi: 10.1002/bit.21965, Biotechnol Bioeng, 101(5), 894-902 (2008)

Storz, G y J.A. Imlay, Oxidative Stress, Curr Opin Microbiol, 2(2), 188-194 (1999)

Takashima, M., T. Sugita, Y. Toriumi y T. Nakase, Cryptococcus tepidarius sp. nov., a thermotolerant yeast species isolated from a stream from a hot-spring area in Japan, doi: 10.1099/ijs.0.004515-0, Int J Syst Evol Microbiol, 59(Pt 1), 181-185 (2009)

Taylor, M.P., K.L. Eley, S. Martin, M.I. Tuffin, S.G. Burton y D.A. Cowan, Thermophilic ethanologenesis: future prospects for second-generation bioethanol production, doi: 10.1016/j.tibtech.2009.03.006, Trends Biotechnol, 27(7), 398-405 (2009) 
Van H.P., J.P. Van Dijken y J.T. Pronk, Effect of specific growth rate on fermentative capacity of baker's yeast, Appl Environ Microbiol, 64(11), 4226-33 (1998)

Vázquez H.J. y O, Da costa, Fermentación alcohólica: Una opción para la producción de energía renovable a partir de desechos agrícolas. Ing. Invest. y tecnol. 8(4), 249-259 (2007)

Virgilio, C.D., T. Hottiger, J. Dominguez, T. Boller y A. Wiemken, The role of trehalose synthesis for the acquisition of thermotolerance in yeast. I. Genetic evidence that trehalose is a thermoprotectant, doi: 10.1111/j.1432-1033.1994.tb19928.x, Eur J Biochem, 219(1-2), 179-186 (1994)

Wartmann, T., A. Krüger, K. Adler, B.M. Duc, I. Kunze y G. Kunze, Temperature-dependent dimorphism of the yeast Arxula adeninivorans Ls3, Antonie Van Leeuwenhoek, 68(3), 215-223 (1995)

Wallace-Salinas, V y M.F. Gorwa-Grauslund, Adaptive evolution of an industrial strain of Saccharomyces cerevisiae for combined tolerance to inhibitors and temperature, doi: 10.1186/1754-6834-6-151, Biotechnol Biofuels, 6(1), 151 (2013)

Webster, D.L y K. Watson, Ultrastructural changes in yeast following heat shock and recovery, doi: 10.1002/yea.320091103, Yeast. 9(11), 1165-1175 (1993)

Weibezahn, J., P. Tessarz, C. Schlieker, R. Zahn, Z. Maglica, S. Lee, H. Zentgraf, E.U. Weber-Ban, D.A. Dougan, F.T. Tsai, A. Mogk y B. Bukau, Thermotolerance requires refolding of aggregated proteins by substrate translocation through the central pore of ClpB, doi:10.1016/j.cell.2004.11.027, Cell, 119(5), 653-665 (2004)

West, T.P y I.I. Kennedy, Isolation of Thermotolerant Yeast Strains for Ethanol Production: A Need for New Approaches, doi: 10.4172/1948-5948, J Microb Biochem Technol 6: 120 (2014)

Zheng, S., M. Yang y Z.Yang, Biomass production of yeast isolate from salad oil manufacturing wastewater, doi:10.1016/j.biortech.2004.09.022, Bioresource technology, 96(10), 1183-1187 (2005)

Zhong, Y., L. Yang, Y. Guo, F. Fang, D. Wang, R. Li, M. Jiang, W. Kang, J. Ma, J. Sun y W. Xiao, Hightemperature cultivation of recombinant Pichia pastoris increases endoplasmic reticulum stress and decreases production of human interleukin-10, doi: 10.1186/s12934-014-0163-7, Microb Cell Factories, 13(1), 163 (2014)

Zoppellari, F y L. Bardi, Production of bioethanol from effluents of the dairy industry by Kluyveromyces marxianus, doi:10.1016/j.nbt.2012.11.017, N Biotechnol, 30(6), 607-613 (2013) 
